Revista Brasileira de Meteorologia, v.26, n.1, 149 - 156, 2011

\title{
CORRELAÇÃO ENTRE AS TEMPERATURAS DA SUPERFÍCIE DO MAR E A QUANTIDADE DA PRECIPITAÇÃO NA ESTAÇÃO CHUVOSA NO NORDESTE DO ESTADO DE PERNAMBUCO
}

\author{
ANA PAULA NUNES SILVA ${ }^{1}$, GEBER BARBOSA DE ALBUQUERQUE MOURA ${ }^{2}$, PEDRO ROGÉRIO \\ GIONGO $^{3}$, SÉRGIO RICARDO RODRIGUES MEDEIROS ${ }^{3}$ \\ ${ }^{1}$ Universidade Federal de Campina Grande, Unidade Acadêmica de Ciências Atmosféricas,Campina Grande, PB. \\ ${ }^{2}$ Universidade Federal Rural de Pernambuco, Departamento de Agronomia, Recife, PB \\ ${ }^{3}$ Escola Superior de Agricultura Luiz de Queiroz, Departamento de Agronomia, Piracicaba, SP \\ apns@ymail.com, geber@depa.ufrpe.br, prgiongo@esalq.usp.br, srrdmede@esalq.usp.br
}

Recebido Fevereiro 2009 - Aceito Junho 2010

\begin{abstract}
RESUMO
O principal objetivo deste trabalho é avaliar o quanto a precipitação no nordeste do Estado de Pernambuco é devido à anomalias de Temperatura da Superfície do Mar (ATSM) do oceano Atlântico e do Pacífico. Para tanto, utilizou-se os totais mensais de precipitação registrados em 15 postos pluviométricos, distribuídos espacialmente ao longo da região estudada dos anos de 1963 a 1992, e ATSM extraídas da versão do Comprehensive Ocean-Atmospheric Data Set (COADS) de 1945 a 1993. Verificou-se que a região estudada possui uma forte correlação positiva com a área de estudo da Bacia do Atlântico Sul, e uma correlação negativa com a Bacia do Pacífico Equatorial. Entretanto, as anomalias na temperatura das águas superficiais da região do Atlântico, por estar adjacente a área analisada, apresentam uma correlação mais forte, ao mesmo nível de significância, que as anomalias do Pacífico. Os resultados mostram que um aquecimento anômalo nesta área do Atlântico pode causar eventos extremos de chuva na costa leste do Nordeste.
\end{abstract}

Palavras-chave: El Niño, gradiente térmico, Oceano Atlântico

\begin{abstract}
CORRELATION BETWEEN SEA SURFACE TEMPERATURE AND INDEX OF RAIN IN THE RAINY STATION IN THE NORTHEAST OF THE PERNAMBUCO STATE

The main objective of this work is to evaluate how much the rain on the northeast of the Pernambuco State is due to the Sea Surface Temperature anomalies (SSTA) of Atlantic and Pacific Ocean. Accumulated monthly rain data registered at 15 rain gauges distributed throughout the studied region during the period from 1963 to 1992 and the 1945 to 1993 SSTA series extracted from the version of Comprehensive Ocean-Atmospheric Data Set (COADS) were used. It was verified that the studied region presents a strong positive correlation with the study area of the South Atlantic Basin and a negative correlation with the Equatorial Pacific Basin. However, the SSTA in the Atlantic region showed a stronger correlation compared to the correlation in the Pacific at the same significance level. This might be due to the proximity of the Atlantic analyzed area. It is suggested that an anomalous heating on this Atlantic area may cause extreme rain events on the east coast of Northeast Region.

Keywords: El-Niño South Oscillation, thermal gradient, Atlantic Ocean.
\end{abstract}




\section{INTRODUÇÃO}

As previsões climáticas são uma ferramenta extremamente útil para ser usada em favor do desenvolvimento sustentável, visto que ajudará no manejo dos recursos naturais e, conseqüentemente, na qualidade de vida das pessoas. A população rural, principalmente a que vive em regiões vulneráveis aos impactos da variabilidade climática, como é o caso do Nordeste do Brasil (NEB), pode usar esta ferramenta para o melhor gerenciamento de seus recursos, que resultará numa ampliação da produção, diminuindo, desta forma, a desigualdade social. Verifica-se assim, que estas previsões são imprescindíveis ao planejamento da sociedade como um todo, do ponto de vista social e econômico.

Walker (1928) observou uma relação entre as secas do NEB e anomalias de temperatura das águas superficiais no Pacífico Equatorial. Daí deu-se início a várias pesquisas que busquem compreender de uma forma mais aprofundada, como anomalias na temperatura da superfície do mar (ATSM) podem causar interferências nos padrões de circulação atmosféricas e, conseqüentemente, afetar o regime pluviométrico em determinadas regiões do globo terrestre.

Há suficientes evidências observacionais de que as condições oceânicas e atmosféricas, nas áreas tropicais no Atlântico e no Pacífico, influenciam fortemente na variabilidade interanual do clima sobre as Américas (Aragão, 1986; Aragão et al., 1994; Hastenrath e Heller, 1977; Moura e Shukla, 1981; Hastenrath et al., 1984; Hastenrath e Greischar, 1993; Moura et al., 2000). Este fato tem motivado diversas pesquisas sobre as relações entre as temperaturas da superfície do mar (TSM) dos Oceanos Pacífico e Atlântico e a precipitação no NEB (Saravanan e Chang, 2000; Pezzi e Cavalcanti, 2001; Giannini, et al., 2004; Andreoli et al., 2004; Kayano e Andreoli, 2004; Ambrizzi et al., 2004; Souza et al., 2004).

Repelli e Nobre (2004) descreveram um sistema estatístico com análises de casos compostos para identificar seqüências críticas de padrões para prever as anomalias de temperatura da superfície do mar nos oceanos tropicais, com ênfase no Atlântico (SIMOC). Segundo esses autores, as estruturas espaciais sobre o Atlântico Tropical (AT), para a estação chuvosa de março a maio, são bem descritas pelas previsões feitas com condições iniciais de setembro a fevereiro.

Ferreira et al. (2004) analisaram a correlação entre a precipitação nas macrorregiões do Estado do Ceará, durante a estação chuvosa e a anomalia de TSM nos oceanos Pacífico Equatorial (índices de El Niño) e AT para 41 anos de dados (1962 a 2002). A correlação linear encontrada entre a precipitação na macrorregião do Sertão Central e Inhamuns, e a anomalia de TSM na bacia sul do Oceano Atlântico Tropical, durante o mês de setembro, foi de $r=0,56$. Foram encontradas altas correlações entre a precipitação na maioria das macrorregiões e o índice de Dipolo entre os meses de setembro e novembro, nas macrorregiões do litoral Norte e Jaguaribana, foi encontrado valor de $r=-0,53$. Também, forte correlação foi encontrada, principalmente, entre a precipitação na macrorregião da Ibiapaba e os índices do Niño3 e Niño3.4 no mês de janeiro, com um valor de -0.50 .

Andreoli e Kayano (2007) estudando a influência que as temperaturas dos Oceanos Atlântico Tropical e o Pacifico exercem sobre o Nordeste do Brasil, perceberam que independente do sinal do ENOS, o Atlântico Tropical (AT) é mais atuante na variabilidade da precipitação na região. $\mathrm{Na}$ ausência de anomalias de Temperatura da Superfície do Mar (ATSMs) significativas no AT, durante a fase inicial e de desenvolvimento do ENOS, em dezembro, janeiro e fevereiro, entenderam que as anomalias na precipitação possivelmente são ocasionadas por padrões de teleconexões associados ao rearranjamento da célula de Walker, e em março, abril e maio, por padrões de teleconexões extratropicais. Simultâneos ENOS e ATSMs significativas no AT atuaram para aumentar ou diminuir as anomalias de precipitação. ATSMs de mesmo sinal no ATS e Pacífico leste enfraqueceram os padrões de precipitação no NEB.

Uma interpretação amplamente aceita é que a variabilidade de TSM do AT seja a forçante dominante das anomalias de precipitação no NEB, enquanto que a influência remota do Pacífico, em determinadas ocasiões pode reforçar estas anomalias, mas em outras ocasiões pode ter efeitos opostos, de modo que estas anomalias sejam enfraquecidas (Uvo et al., 1998; Pezzi e Cavalcanti, 2001; Ambrizzi et al., 2004; Souza et al., 2004, Andreoli e Kayano, 2007).

Moura et al (2009) correlacionou a precipitação do período chuvoso do setor leste do NEB com ATSM's dos oceanos Atlântico e Pacífico, defasadas no tempo em até 10 períodos (defasagens de -10), e identificaram que o Oceano Atlântico possui maior influência sobre o regime pluviométrico do setor leste do Nordeste, indicando que anomalias positivas neste cooperam em precipitações acima da média, e ATSM negativas inibem a precipitação na região estudada.

Neste trabalho objetivou-se estudar a auto-correlação da precipitação na estação chuvosa (março a julho) da região nordeste do Estado de Pernambuco, com anomalias de temperatura da superfície do mar (TSM) nos oceanos Atlântico e Pacífico, e identificar áreas nos dois oceanos que influenciam a qualidade do regime de chuvas na região estudada.

\section{MATERIAL E MÉTODOS}

A área de estudo compreende uma faixa que vai do litoral e adentra o Estado de Pernambuco por aproximadamente 
$200 \mathrm{~km}$, compreendida entre os paralelos de $7^{\circ} 15^{\prime}$ e $8^{\circ}$ e $30^{\prime}$ de latitude sul, e entre os meridianos de $34^{\circ} 48^{\prime}$ e $36^{\circ} 49^{\prime}$ de longitude a oeste de Greenwich.

A região selecionada para o estudo corresponde a uma área de aproximadamente $13.511,54 \mathrm{Km}^{2}$ ou $14 \%$ do território pernambucano, que compreende 72 municípios.

Neste trabalho utilizaram-se totais mensais de precipitação registrados em 15 postos pluviométricos distribuídos espacialmente ao longo da região estudada, a partir de dados obtidos no Instituto de Pesquisas Agropecuárias - IPA. Estes dados abrangem um período de observação compreendido entre 1963 a 1992. O período chuvoso foi determinado de acordo com as sequiências dos quatro meses mais chuvosos do ano observado, através da média de cada mês para o período do estudo, encontrando-se assim apenas uma quadra chuvosa, visto que a região escolhida para o estudo é homogênea no que concerne ao regime pluviométrico. Desta forma, o quadrimestre chuvoso encontrado inicia-se no mês de abril e segue até o mês de julho.

A distribuição espacial dos postos pluviométricos envolvidos na pesquisa é mostrada na Figura 1.

Para a realização da análise, procedeu-se a normalização da precipitação total no período chuvoso em cada posto pluviométrico, calculando-se a partir daí o desvio normalizado médio para cada posto por ano $\left(\bar{P}_{k i}\right)$, mediante o emprego da Equação 1.

$$
\bar{P}_{k i}=\frac{P_{i}-\mu_{1}}{\sigma_{1}}
$$

Onde:

$P_{i}$ - precipitação na quadra chuvosa no ano i;

$\mu_{1}$ - média climatológica da quadra chuvosa no período estudado; e,

$\sigma_{1}$ - desvio padrão (por cada posto) para o conjunto de dados analisado.

A precipitação normalizada $(\overline{P N})$ para o quadrimestre mais chuvoso para o conjunto de dados foi calculada pela expressão:

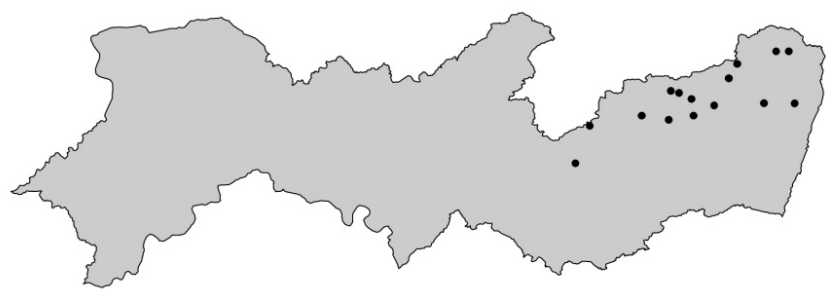

Figura 1 - Distribuição espacial dos postos pluviométricos utilizados no estudo

$$
\overline{P N}_{i}=\frac{\bar{P}_{k i}-\mu_{2}}{\sigma_{2}}
$$

onde: $\mu_{2}$ - média das precipitações normalizadas de todos os postos; e, $\sigma_{2}$ - desvio padrão das precipitações normalizadas dos postos

As anomalias mensais de TSM foram extraídas da mais nova versão do Comprehensive Ocean-Atmospheric Data Set (COADS), compilada por Da Silva et al. (1994), a qual possui a resolução em cada ponto na grade $1^{\circ} \mathrm{X} 1^{\circ}$ de latitude e longitude para o período de 1945 a 1993, estas foram observadas sobre os oceanos Pacifico e Atlântico, e calculadas as médias das anomalias de abril a julho.

Para estudar as correlações lineares entre a precipitação com as anomalias de TSM, as análises fundamentaram-se na série dos totais de precipitação de abril a julho e anomalias de TSM para o período de 1963 a 1992.

Os sinais analisados de anomalias dos oceanos foram obtidos das áreas que tiveram maiores correlações com o regime pluviométrico na região analisada (Figura 3, "defasagem 0"), áreas coincidentes com Moura et al. (2009). Desta forma, o gradiente do AT foi caracterizado pela diferença da anomalia de temperatura na Bacia Norte pela anomalia da Bacia Sul, obtidas das seguintes áreas:

Área $1: 45,5^{\circ} \mathrm{W}$ a $25,0^{\circ} \mathrm{W}$ e $45,0^{\circ} \mathrm{N}$ a $59,5^{\circ} \mathrm{N}$.

Área $2: 46,7^{\circ} \mathrm{W}$ a $37,5^{\circ} \mathrm{W}$ e $6,3^{\circ} \mathrm{N}$ a $12,0^{\circ} \mathrm{N}$.

Área 3: $10,0^{\circ} \mathrm{W}$ a $14,3^{\circ} \mathrm{E}$ e $14,0^{\circ} \mathrm{S}$ a $3,0^{\circ} \mathrm{S}$.

O sinal da ATSM do Pacífico foi obtido pelas anomalias registradas nas seguintes áreas:

Área $1: 80,0^{\circ} \mathrm{W}$ a $100,0^{\circ} \mathrm{W}$ e $9,0^{\circ} \mathrm{S}$ a $15,0^{\circ} \mathrm{S}$.

Área 2: $80,0^{\circ} \mathrm{W}$ a $100,0^{\circ} \mathrm{W}$ e $0^{\circ} \mathrm{S}$ a $9,0^{\circ} \mathrm{S}$.

Área 3: $100,0^{\circ} \mathrm{W}$ a $140,0^{\circ} \mathrm{W}$ e $10,0^{\circ} \mathrm{N}$ a $10,0^{\circ} \mathrm{S}$.

Área 4: $140,0^{\circ} \mathrm{W}$ a $180,0^{\circ} \mathrm{W}$ e $10,0^{\circ} \mathrm{N}$ a $10,0^{\circ} \mathrm{S}$.

Estas áreas do Pacífico foram selecionadas, pois de acordo com Moura e Shukla (1981) o regime pluviométrico sobre o NEB seja o estabelecimento de uma circulação térmica direta local, que modifica a circulação normal de Hadley, a qual tem seu ramo ascendente em torno de $10 \mathrm{~N}$ e seu ramo descendente sobre o NEB, Amazônia oriental e regiões oceânicas vizinhas.

Realizou-se correlações lineares entre os desvios normalizados do grupo, e anomalias de TSM em cada ponto de grade até o defasagem -4, isto é, a série de precipitação normalizada (média abril a julho) foi correlacionada com as séries de anomalias (médias de 4 meses) de TSM do mesmo período até os 4 períodos anteriores (defasagem -1 até defasagem -4) para os dois oceanos. O primeiro período (defasagem 0 ) corresponde ao período chuvoso de abril a julho, o segundo período (defasagem -1) corresponde aos meses de março a 
Tabela 1 - Retardos de ATSM com relação à precipitação normalizada

\begin{tabular}{ccc}
\hline Defasagem & $\begin{array}{c}\text { Índices de } \\
\text { Precipitação }\end{array}$ & ATSM \\
\hline Defasagem 0 & Abril a julho & Abril a Julho \\
Defasagem -1 & Abril a julho & Março a Junho \\
Defasagem -2 & Abril a julho & Fevereiro a Maio \\
Defasagem -3 & Abril a julho & Janeiro a Abril \\
& & \\
Defasagem -4 & Abril a julho & Novembro a Março \\
\hline
\end{tabular}

junho. Conseqüentemente, os demais períodos de defasagens negativas são correspondentes a grupos de 4 meses, seguindo uma seqüência onde se subtrai sempre um mês. A Tabela 1 mostra como as defasagens foram construídas. Para verificar a significância das correlações foi realizado o teste t-Tukey, ao nível de significância estatística $\mathrm{p}<0,05$.

\section{RESULTADOS E DISCUSSÃO}

\subsection{Variabilidade interanual pluviométrica do setor leste do NEB}

Na Tabela 2 são apresentados os resultados das normalizações, correspondentes aos anos de 1963 a 1992. Na Figura 2 verificam-se as anomalias de TSM para o Oceano Pacífico e Atlântico. Os eventos de anomalias positivas no

Tabela 2 - Precipitação normalizada para os postos pluviométricos do Nordeste de Pernambuco

\begin{tabular}{cccc}
\hline ANO & $\overline{P N}$ & ANO & $\overline{P N}$ \\
\hline 1963 & $-0,8687$ & 1978 & 0,9878 \\
1964 & 0,176 & 1979 & $-0,5908$ \\
1965 & 0,2778 & 1980 & $-1,3366$ \\
1966 & 1,0836 & 1981 & $-1,9902$ \\
1967 & 0,4305 & 1982 & $-0,2962$ \\
1968 & $-0,8373$ & 1983 & $-1,5073$ \\
1969 & 0,5843 & 1984 & 1,2062 \\
1970 & $-0,3222$ & 1985 & 1,1123 \\
1971 & 0,084 & 1986 & 0,4277 \\
1972 & $-0,5535$ & 1987 & $-0,5536$ \\
1973 & $-0,1299$ & 1988 & 0,2435 \\
1974 & 0,6896 & 1989 & 0,6788 \\
1975 & 2,1137 & 1990 & $-0,5123$ \\
1976 & $-0,9978$ & 1991 & $-0,9188$ \\
1977 & 2,1176 & 1992 & $-0,7984$ \\
\hline
\end{tabular}

Pacífico Equatorial (El Niño) estão de acordo com Rasmusson e Carpenter (1983), Ropelewski e Halpert (1987, 1989). Confrontando-se a Tabela 2 com a Figura 2, percebe-se que nos anos de 1966, 1975, 1977, 1978, 1984 e 1985, os índices de precipitação ficaram acima da média, indicando a presença da fase negativa do Padrão de Dipolo observados nos anos de 1964, 1974 e 1985. Também se pode observar que os anos de 1976, 1980, 1981 e 1983 obtiveram índices de precipitação bem abaixo da média climatológica. Neste último há uma forte tendência de que o baixo índice de precipitação está relacionado com a ocorrência do El-Niño e uma neutralidade das anomalias do Atlântico.

Verifica-se também, a existência de anos em que as condições oceânicas estavam favoráveis e as chuvas observadas foram próximas à da média. Como por exemplo, no ano de 1971, as águas do Atlântico Sul estavam mais quentes (favorecendo a precipitação) e as águas do Pacífico Equatorial estavam com temperaturas abaixo da média, caracterizando ano de influência da fase fria do ENOS (La-Niña), desfavorecendo o regime pluviométrico na região analisada. Entretanto, as precipitações na região de estudo estiveram em torno da média durante a estação chuvosa desse ano.

\subsection{Correlações entre os desvios normalizados de precipitação e as anomalias de TSM}

As primeiras análises feitas consistiram em calcular os coeficientes de correlação entre os Desvios Normalizados e os campos globais de anomalias de TSM, para cada ponto de grade dos Oceanos Pacífico e Atlântico, para a "defasagem 0" e as demais defasagens.

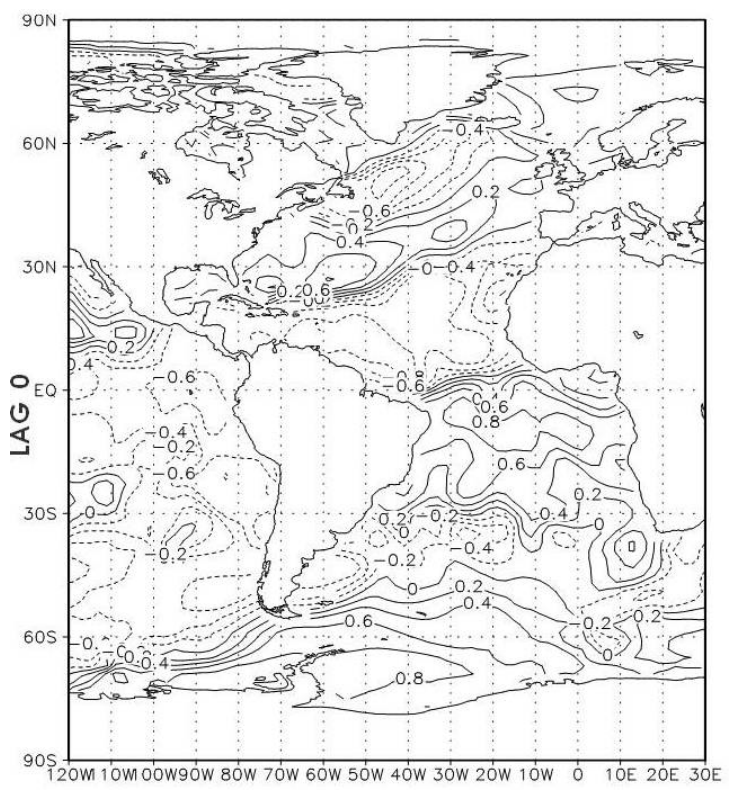

Figura 2 - Anomalias de temperaturas das bacias estudadas para o período analisado (1963 a 1992) 
Na Figura 3 são apresentados os mapas globais das correlações entre os índices de precipitação e anomalias de TSM. Para a "defasagem 0" é possível observar que no oceano Pacífico, na área do El Niño 2, há uma forte correlação negativa, apresentando um índice de determinação maior que 0,5 (com significância estatística de $\mathrm{p}<0,05$ ). Nota-se também que na Bacia do Atlântico Norte há uma alta correlação amostral negativa, (com significância estatística de $\mathrm{p}<0,05$ ), resultando em elevado coeficiente de determinação. Já na Bacia do Atlântico Sul, o fator de correlação apresentou um alto valor positivo, revelando grau de determinação maior que 0,64.

Ainda para a "defasagem 0" pode-se verificar também $\mathrm{o}$ aparecimento de correlações tanto negativas, entre o equador até $15^{\circ} \mathrm{S}(\mathrm{r}>0,4$, com significância estatística de $\mathrm{p}<0,05)$, como positivas, valores entre $10^{\circ}-30^{\circ} \mathrm{S}$ e aproximadamente $140^{\circ} \mathrm{W}$
( $r>0,6$, com significância estatística de $p<0,05)$ no Pacífico. A precipitação na área estudada mostrou uma forte correlação negativa com a área de El-Niño 1+2, chegando a obter um grau de correlação maior que 0,6 .

Para todas as defasagens verificaram-se setores de correlações negativas e positivas, respectivamente ao norte e sul do Atlântico Equatorial, compreendidas na área do Dipolo, Moura e Shukla (1981), com maiores correlações positivas $(r>0,8$ com significância estatística de $\mathrm{p}<0,05)$.

As correlações da precipitação com o oceano Pacífico foram negativas para todas as defasagens estudadas, implicando, assim, uma influência negativa nas chuvas do setor nordeste do Estado de Pernambuco, como era de se esperar, pois nos episódios El-Niño as águas quentes nesta bacia modificam a circulação de Walker de forma a provocar subsidência troposférica anômala
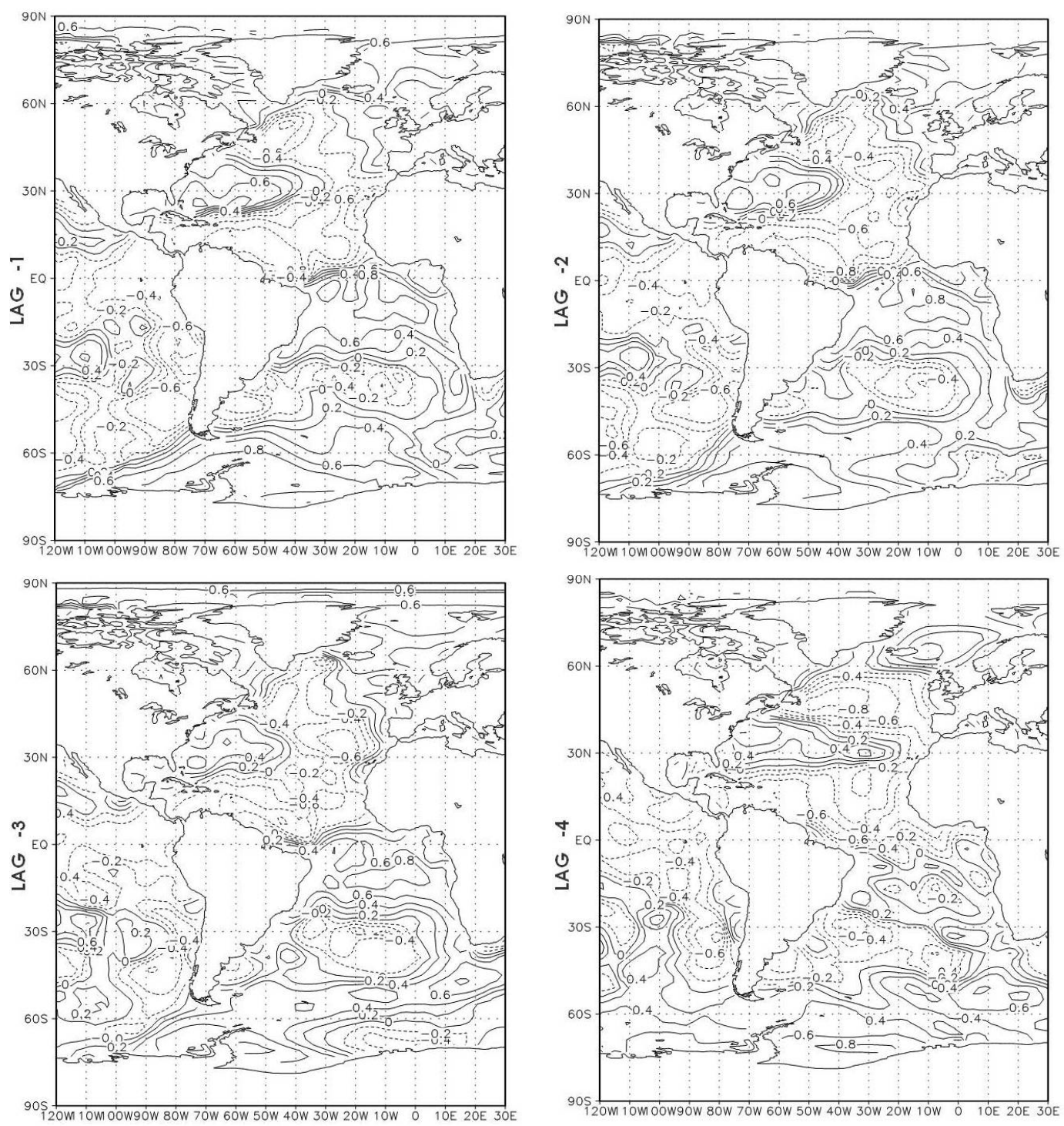

Figura 3 - Correlações entre a Precipitação Normalizada no Nordeste do Estado de Pernambuco (média de março a julho) e anomalias de TSM da defasagem 0 a defasagem -4 (de 1963 a 1992) com significância estatística $p<0,05$ 
e reduzir as chuvas no NEB. Como observado por Kousky e Cavalcanti (1984), Kayano et al. (1988) e Ropelewski e Halpert (1987 e 1989), os quais verificaram que sob condições de El Niño, a circulação de Walker é deslocada para leste, de tal maneira que o seu ramo ascendente localiza-se sobre o oceano Pacífico central e leste onde a convecção é intensificada, e seu ramo descendente localiza-se sobre o e Atlântico Tropical (AT) adjacente, onde a convecção é inibida. Este fato tem levado a associar fenômenos de seca no NEB, em anos onde a TSM do Pacífico Equatorial apresenta anomalias positivas.

Por sua vez, durante os episódios de La Niña, a influência do Pacífico é positiva com relação às chuvas no nordeste de Pernambuco, com aceleração dos movimentos verticais ascendentes, intensificação dos sistemas meteorológicos atuantes, formação de mais nuvens e aumento da precipitação.

No Atlântico tropical Sul, junto à costa oeste da África, identificou-se uma região com correlações positivas e significativas variando de 0,2 a 0,8 . Conforme as defasagens se aproximam do período chuvoso, esta região de correlações positivas move para oeste até alcançar a área adjacente à costa nordestina. Esse movimento também foi verificado por Moura et al. (2009) para todo o setor leste do nordeste.

Foram encontradas correlações negativas e significativas junto à Terra Nova (Atlântico Norte) variando de 0,4 a 0,6 para as defasagens analisadas. Esse resultado pode está relacionado com a teleconexão encontrada por Namias (1972). Ele mostrou que a precipitação de fevereiro a maio em Quixeramobim, no Ceará, é correlacionada com a circulação da atmosfera na região da Terra Nova/Groelândia, durante as estações precedentes ao inverno e à primavera no Hemisfério Norte. Essa relação climática está associada com a variação na intensidade e posição da ZCIT, e com a teleconexão conhecida como "Pacific North America" (PNA), que também está relacionada com o fenômeno ENOS. Quando existe situação de bloqueio na Terra Nova e Groelândia, o anticiclone dos Açores se intensifica movendo a ZCIT mais para o sul e provocando chuvas no NEB. Por outro lado, quando existe forte atividade ciclônica na Terra Nova/ Groelândia, o anticiclone fica mais fraco, a ZCIT fica mais ao norte do que o normal e então ocorre seca no NEB.

Como pode ser observada, a partir desses resultados, a influência da distribuição de anomalias de TSM sobre as chuvas do setor leste do NEB sugere que, para as estações aqui analisadas, a influência maior é do Atlântico sul, com correlações positivas em sua maioria. Isso indica que águas quentes, nessa região do oceano, afetam positivamente as chuvas, e água mais fria, negativamente. Haja vista que, águas mais quentes no AT Sul provocará mais evaporação da área adjacente à costa do NEB, devido ao maior fluxo de calor latente na área tropical, que leva umidade aos altos níveis e esta massa quando chega ao continente, se condensa em forma de precipitação.

\section{CONCLUSÕES}

As maiores correlações entre a estação chuvosa no nordeste do Estado de Pernambuco e anomalias de temperatura da superfície do mar foram encontradas no Oceano do Atlântico. A influência da distribuição das anomalias de TSM sobre as chuvas no Nordeste de Pernambuco está diretamente relacionada com áreas do Atlântico Tropical Sul e áreas do Pacífico Equatorial. Águas quentes na Bacia do Atlântico Sul afetam positivamente as chuvas, enquanto que águas mais frias, impactam negativamente a estação chuvosa nesta região do Estado. Em relação à região do Pacífico, quando as águas apresentaram temperatura abaixo da média há um acréscimo na precipitação no setor nordeste do Estado.

Observou-se uma área de correlações positivas no Golfo de Guiné, que se move para oeste à medida que as defasagens vão diminuindo, até alcançar a área adjacente à costa nordestina. Este fato ocorre devido a esta área estar em latitudes próximas às da região continental analisada, fazendo com que a água evaporada nesta região ascenda aos médios níveis da atmosfera e, devido a sistemas de grande (distúrbios ondulatórios de leste) e de mesoescala (sistema de brisa marítima) este ar úmido é levado ao continente, causando chuva na área estudada.

No Atlântico norte, as correlações negativas verificadas junto a Terra Nova/Groelândia, variando de 0,4 a 0,6 parecem ser relacionadas com as atividades de bloqueio (ou fortalecimento ciclônico), que induzem a chuvas intensas (ou secas) no NEB, através do fortalecimento (enfraquecimento) da alta dos Açores com conseqüente deslocamento para sul (ou para norte) da ZCIT.

De acordo com o IPCC (2007) em cenários de mudanças climáticas globais, a região tropical é a mais afetada por anomalias positivas na temperatura da superfície do mar. Portanto, estudar a temperatura dos oceanos compreendidos nesta faixa do Globo, com o objetivo de auxiliar na quantificação da precipitação em uma determinada região, possibilita determinada com antecedência os índices de chuva. Este fato pode auxiliar os órgãos de defesa civil a se programar para minimizar os impactos de desastres de cunho sócio-ambiental, que venham acontecer nas áreas afetadas por extremos de chuva, principalmente nos centros urbanos, onde o crescimento desordenado, associado a eventos excessos de índices pluviais é o fator gerador das grandes catástrofes ambientais que assolam estas áreas.

\section{REFERÊNCIAS BIBLIOGRÁFICAS}

AMBRIZZI, T.; SOUZA, E. B.; PULWARTY, R. S. The Hadley and walker regional circulations and associated ENSO impacts on south american seasonal rainfall. In: the hadley circulation: present, past and future, Diaz H.F. and Bradley R.S. (Eds.). Kluwer Publishers, Chapter 7, p. 203-235, 2004. 
ANDREOLI, R. V.; KAYANO, M. T. A importância relativa do Atlântico tropical sul e Pacífico leste na variabilidade de precipitação do Nordeste do Brasil. Revista Brasileira de Meteorologia, v. 22, n. 1, p. 63-74, 2007.

ANDREOLI, R. V.; KAYANO, M. T.; GUEDES, R. L.; OYAMA, M. D.; ALVES, M. A. S. A influência da temperatura da superfície do mar dos oceanos Pacífico e Atlântico na variabilidade de precipitação em Fortaleza. Revista Brasileira de Meteorologia, v. 19, n. 3, p. 337344, 2004.

ARAGÃO, J. O. R. A general circulation model investigation of the atmospheric response to El Niño. National Center for Atmospheric Research (NCAR)/CT-100: Boulder-CO, U.S.A. 144p. 1986.

ARAGÃO, J. O. R.; ROUCOU, P.; HARZALLAH, A.; Fontaine, B.; Janicot, S. Variabilité atmosphérique sur le Nordeste brésilien dans le modèle de circulation générale du LMD (1970-1988). In: Publications de l'Association Internationale de Climatologie, Grécia. Resumos... Panglotis Maheras n.7. 1994. p.432-438.

DA SILVA, A. M.; YOUNG, C. C.; LEVITUS, S. Atlas of surface marine data 1994. v.1: Algorithms and procedures. NOAA ATLAS NESDIS 6, Washington, 88pp., 1994.

FERREIRA, F. F.; ALVES, J. M. B.; COSTA, A. A. Um estudo dos impactos das Temperaturas da Superfície do Mar (TSM) em macrorregiões, pluviometricamente homogêneas, no Estado do Ceará. Revista Brasileira de Agrometeorologia, v. 12, n. 1, p. 193-204, 2004.

GIANNINI, A.; SARAVANAN, R.; CHANG, P. the preconditioning role of Tropical Atlantic Variability in the development of the ENSO teleconnection: implications for the prediction of Nordeste rainfall. Climate Dynamics, v. 22, p. 839-855, doi: 10.1007/s00382-004-0420-2, 2004.

HASTENRATH, S.; GREISCHAR, L. Further work on the prediction of Northeast Brazil rainfall anomalies. Journal of Climate, v. 6, p. 743-758, 1993.

HASTENRATH, S.; HELLER, L. Dynamics of climatic hazards in Northeast Brazil. Quarterly Journal of the Royal Meteorological Society, Berkshire, v. 103, p. 77-92, 1977.

HASTENRATH, S.; WU, M. C.; CHU, P. S. Towards the monitoring and prediction of Northeast Brazil droughts. Quarterly Journal of the Royal Meteorological Society, v.110, p.411-425, 1984.

IPCC. Summary for Policymakers. In: Climate Change 2007: The Physical Science Basis. Contribution of Working Group I to the Fourth Assessment Report of the Intergovernmental Panel on Climate Change [Solomon, S., D. Qin, M. Manning, Z. Chen, M. Marquis, K.B. Averyt, M.Tignor and H.L. Miller (eds.)]. Cambridge University Press, Cambridge, United Kingdom and New York, NY, USA. 2007.
KAYANO, M. T.; ANDREOLI, R. V. Decadal variability of northern northeast Brazil rainfall and its relation to tropical sea surface temperature and global sea level pressure anomalies. Journal Geophysical Research, v. 109, n. C11011, 8 pp., 2004.

KAYANO, M. T.; RAO, V. B.; MOURA, A. D. Tropical circulations and the associated rainfall anomalies during two contrasting years. Journal of Climatology, v. 8, n. 5, p. 477-488, 1988.

KOUSKY, V. E.; CAVALCANTI, I. F. A. Eventos Oscilação do Sul - El Niño: Características, evolução e anomalias de precipitação. Ciência e Cultura, v. 36, n. 11, p. 18881899, 1984.

MOURA, A. D.; SHUKLA, J. On the dynamics of the droughts in Northeast Brazil: Observations, theory and numerical experiments witn a general circulations model. Journal of the Atmospheric Science, v. 38, n. 12, p. 2653-2673, 1981.

MOURA, G. B. A.; ARAGÃO, J. O. R.; LACERDA, F. F.; PASSAVANTE, J. Z. O. Relação entre a precipitação no setor leste do Nordeste do Brasil e a temperatura da superfície nos oceanos Atlântico (área do Dipolo) e Pacífico. Revista Brasileira de Engenharia Agrícola e Ambiental, Campina Grande, v. 4, n. 2, p. 247-251, 2000.

MOURA, G. B. A.; ARAGÃO, J. O. R.; MELO, J. S. P.; SILVA, A. P. N.; GIONGO, P. R.; LACERDA, F. F. Relação entre a precipitação do leste do Nordeste do Brasil e a temperatura dos oceanos. Revista Brasileira de Engenharia Agrícola e Ambiental, v. 13, p. 462-469, 2009.

NAMIAS, J. Influence of Northern Hemisphere general circulation on drought in Northeast Brazil. Tellus, v. 24, n. 4, p. 336-343, 1972.

PEZZI, L. P.; CAVALCANTI, I. F. A. The relative importance of ENSO and tropical Atlantic sea surface temperature anomalies for seasonal precipitation over South America: a numerical study. Climate Dynamics, v. 17, p. 205-212, 2001.

RASMUSSON, E. M.; CARPENTER, T. H. The relationship between eastern equatorial Pacific sea temperature and rainfall over Índia and Sri Lanka. Monthly Weather Review, v. 111, p. 517-528, 1983

REPELLI, C. A.; NOBRE, P. Statistical prediction of seasurface temperature over the tropical Atlantic. International Journal of Climatology. v. 24, p. 45-55, 2004.

ROPELEWSKI, C. F.; HALPERT, M. S. Global and regional scale precipitation patterns associated with El Niño/Southern Oscillation. Monthly Weather Review, v. 115, p. 16061626, 1987.

ROPELEWSKI, C. F.; HALPERT, M. S. Precipitation patterns associated with high index phase of Southern Oscillation. Journal of Climate, v. 2, p. 268-284, 1989.

SARAVANAN, R.; CHANG, P. Interaction between Tropical 
Atlantic variability and El Niño-southern oscillation. Journal of Climate, v.13, n.13, p. 2177-2194, 2000.

SOUZA, E. B.; AMBRIZZI, T.; COELHO, C. A. S. Two episodes with reversed impacts on the regional precipitation of the northeastern South America. Meteorologica, v.29, n. 1-2, p. 5-16, 2004.

UVO, C. R. B.; REPELLI, C.; ZEBIAK, S. E.; KUSHNIR, $Y$. The relationships between Tropical Pacific and Atlantic
SST and Northeast Brazil monthly precipitation. Journal of Climate, v.11, p. 551-562, 1998.

WALKER, G. T. Ceará (Brazil) famines and the general air movement. Beitrage zur Physik der freien Atmosphare, v.14, p.88-93, 1928. 